5. Raphael Salem, On sets of multiplicity for trigonometrical series, Amer. J. Math. vol. 64 (1942) pp. 531-538.

6. Yu. A. Sreider, The structure of maximal ideals in rings of measures with convolution, Amer. Math. Soc. Translation, no. 81, Providence, 1953.

7. Antoni Zygmund, Trigonometric series, 2d ed., vol. I, Cambridge University Press, 1959.

UNIVERSITY OF WISCONSIN

\title{
ARITHMETIC PROPERTIES OF CERTAIN POLYNOMIAL SEQUENCES
}

BY L. CARLITZ

Communicated by G. B. Huff, March 23, 1960

Consider the sequence of polynomials $\left\{u_{n}(x)\right\}$ that satisfy the recurrence

$$
u_{n+1}(x)=(x+a(n)) u_{n}(x)+b(n) u_{n-1}(x),
$$

where $a(n), b(n)$ are polynomials in $n$ (and possibly some additional indeterminates) with integral coefficients. Moreover it is assumed that

$$
u_{0}(x)=1, \quad u_{1}(x)=a(0), \quad b(0)=0 .
$$

The sequence $\left\{u_{n}(x)\right\}$ is uniquely determined by (1) and (2).

The writer [1, Theorem 1] has proved that if $m \geqq 1, r \geqq 1$, then $u_{n}(x)$ satisfies the congruence

$$
\sum_{s=0}^{r}(-1)^{s}\left(\begin{array}{l}
r \\
s
\end{array}\right) u_{n+s m}(x) u_{(r-s) m}(x) \equiv 0\left(\bmod m^{r_{1}}\right),
$$

for all $n \geqq 1$, where

$$
r_{1}=[(r+1) / 2],
$$

the greatest integer $\leqq(r+1) / 2$. In the present paper it is proved that $u_{n}(x)$ satisfies the simpler congruence

$$
\sum_{s=0}^{r}(-1)^{s}\left(\begin{array}{l}
r \\
s
\end{array}\right) u_{n+s m}(x) u_{m}^{r-s}(x) \equiv 0\left(\bmod m^{r_{1}}\right),
$$

where again $r_{1}$ is defined by (4). Also it is shown that (5) implies

$$
\sum_{s=0}^{r}(-1)^{s}\left(\begin{array}{l}
r \\
s
\end{array}\right) u_{n+s m}(x) u_{k+(r-s) m}(x) \equiv 0\left(\bmod m^{r_{1}}\right),
$$


for all $n \geqq 0, k \geqq 0$; for $k=0$, (6) evidently reduces to (3). Indeed if we put

$$
U_{k}^{(r)}=U_{n_{1}}^{(r)}, \cdots, n_{k}(x)=\sum_{s_{1}+\cdots+s_{k}=r} \frac{r !}{s_{1} ! \cdots s_{k} !} \lambda_{1}^{s_{1}} \cdots \lambda_{k}^{s_{k}} \prod_{j=1}^{k} u_{n_{j}+s_{j} m}(x),
$$

where $\lambda_{1}, \cdots, \lambda_{k}$ are rational numbers that are integral $(\bmod m)$ and such that

$$
\lambda_{1}+\cdots+\lambda_{k} \equiv 0(\bmod m),
$$

then it is shown that

$$
U_{k}^{(r)} \equiv 0\left(\bmod m^{r_{1}}\right)
$$

for all $n_{1}, \cdots, n_{k} \geqq 0$.

We remark that the congruence (7) was suggested by certain congruences for the Bernoulli numbers that were obtained by Vandiver [2].

There are numerous applications of (5). In particular we mention the following which is related to elliptic functions. The Stieltjes formula $[3$, p. 374]

$$
\int_{0}^{\infty} \operatorname{sn}\left(u, k^{2}\right) e^{-x u} d u=\frac{1}{x^{2}+a-} \frac{1 \cdot 2^{2} \cdot 3 k^{2}}{x^{2}+3^{2} a-x^{2}+5^{2} a-5 k^{2}} \cdots,
$$

where $a=1+k^{2}$, suggests the consideration of the polynomials $f_{n}(x)$ defined by

(8) $f_{n+1}(x)=\left(x+(2 n+1)^{2} a\right) f_{n}(x)-(2 n-1)(2 n)^{2}(2 n+1) k^{2} f_{n-1}(x)$, together with $f_{0}(x)=1, f_{1}(x)=x+a$. Since (8) is of the form (1), it follows that these polynomials satisfy (5). Similar results hold for the polynomials associated in like manner with the integrals

$$
x \int_{0}^{\infty} s n^{2}\left(u, k^{2}\right) e^{-x u} d u, \quad \int_{0}^{\infty} c n\left(u, k^{2}\right) e^{-x u} d u, \quad \int_{0}^{\infty} d n\left(u, k^{2}\right) e^{-x u} d u .
$$

We remark that (8) implies

$$
\sum_{n=0}^{\infty} f_{n}\left(x^{2}\right) \frac{s n^{2 n+1} u}{(2 n+1) !}=\frac{\sinh x u}{x} .
$$

We show also that if $p=2 w+1$ is an odd prime then $f(x) \equiv \bar{f}(x)(\bmod$ $p)$, where

$$
\bar{f}_{p}(x)=x\left\{x^{w}-C_{p}\left(k^{2}\right)\right\}^{2}
$$

and 


$$
C_{p}\left(k^{2}\right)=(-1)^{w} \sum_{s=0}^{w}\left(\begin{array}{c}
w \\
s
\end{array}\right)^{2} k^{28} .
$$

Thus (5) reduces to

$$
\sum_{s=0}^{r}(-1)^{s}\left(\begin{array}{c}
r \\
s
\end{array}\right) f_{n+s p}(x) f_{p}^{r-s}(x) \equiv 0\left(\bmod p^{r_{1}}\right),
$$

where $\bar{f}_{p}(x)$ is defined by (9) and (10).

\section{REFERENCES}

1. L. Carlitz, Congruence properties of the polynomials of Hermite, Laguerre and Lagrange, Math. Z. vol. 59 (1954) pp. 474-483.

2. H. S. Vandiver, Note on a certain ring congruence, Bull. Amer. Math. Soc. vol. 43 (1937) pp. 418-423.

3. H. S. Wall, Analytic theory of continued fractions, New York, 1948.

DUKE UNIVERSITY 